\title{
Synthesis of Tetrazole Regioisomers of Biphenyl as ACE Inhibitors
}

\author{
Gangadhar Y. Meti, Ravindra R. Kamble*, Mahadev N. Kumbar, Saba Kauser J. Shaikh, Atulkumar \\ A. Kamble, Hirehalli. C. Devarajegowda, Praveen K. Bayannavar \\ ${ }^{1}$ Department of Studies in Chemistry, Karnatak University, Pavate Nagar, Dharwad - 580003, India \\ ${ }^{2}$ Department of Physics, Yuvaraja College, University of Mysore, Mysuru - 570005, India \\ Email: ravichem@kud.ac.in
}

\begin{abstract}
Novel angiotensin converting enzyme (ACE) inhibitors were designed based on the structure of commercially available sartans and synthesized by N-alkylation of 5-substituted-1Htetrazole with 4'-(bromomethyl)-biphenyl-2-carbonitrile gave 1,5- and 2,5- regioisomers of tetrazole. The nitrile group appended to biphenyl nucleus was converted into tetrazole and later the tetrazole was ring transformed into 1,3,4-oxadiazole. The synthesized compounds were screened for the ACE inhibition and some of the compounds exhibited significant results.
\end{abstract}

Keywords: Biphenyl, tetrazole, N-alkylation, regioisomers, 1,3,4-Oxadiazole, ring transformation, ACE inhibition

\section{Introduction}

All the cardiovascular diseases in the present era are caused mainly by "lifestyle disorder", a term coined to redefine hypertension. Although the causes of cardiovascular disease are many, hypertension stands to be the crucial one. A recent study suggests that hypertensive patients are more prone to develop type II diabetes [1]. Thus, these chronic diseases coexist, with increasing risk of coronary artery disease, kidney damage, vision problems, enlarging of heart, stroke and heart failure. Many drugs have been developed to treat such chronic diseases with reduced risk of side effects. Thus, the efficacy of hypertensive medications involves many etiologies and disease mechanisms to treat the hypertensive related chronic disease [2].

The first line therapy for the treatment of hypertension is angiotensin converting enzyme (ACE) inhibitors and angiotensin II receptor blockers. The biphenyl-tetrazole is the key substrate [3-5], which belongs to a group of angiotensin II-receptor antagonists generally referred to as "sartans". These include Losartan, Valsartan and Candesartan which are useful in the treatment of hypertension, heart diseases, and bladder diseases and work by blocking the action of angiotensin II on its receptor (Figure 1). Tetrazole is a heterocyclic moiety that functions as a carboxylic acid bioisostere with greater metabolic stability. It forms two hydrogen bonds with peptide residues in a biological target site which facilitates the binding interaction [6]. The negative charge of the tetrazolic anion is delocalized over all four nitrogens which play a major role in the $\operatorname{cog} \mathrm{P}$ value and thus resulting in better oral bioavailability and cell penetration. It also exhibits many biological activities like antihypertensive $[7,8]$, anti-inflammatory [9], endocannabinoid metabolism inhibitor, antibiotic [10] and anticonvulsant [11]. 1,3,4-Oxadiazole is a biologically active nucleus which shows a broad spectrum of pharmacological activities such as antimicrobial [12], antiprotozoal [13], anti-inflammatory [14], anti-tubercular [15] etc. Thus, the combination of two biological active moieties enhances the biological activities of the novel template.

Considering all the above observations, we intended to synthesize novel scaffolds by N-alkylation of 5substituted-1 $H$-tetrazole 2 a-d with 4'-(bromomethyl)-biphenyl-2-carbonitrile 1 , there by affording two tetrazole regioisomers. Both the separated isomers 3a-d and 4a-d containing a nitrile group were converted into the corresponding tetrazole derivatives (5a-d and 6a-d). These were further ring transformed into 1,3,4-oxadiazoles 7a-d and 8a-d respectively in presence of acetic anhydride as shown in Scheme 1. All titled compounds were also subjected to the in vitro ACE inhibitions assay. 


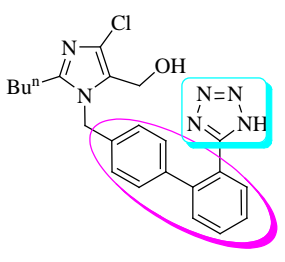

Losartan

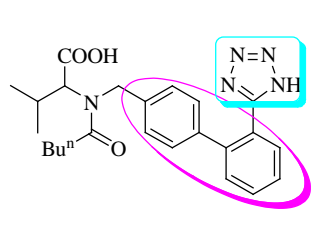

Valsartan

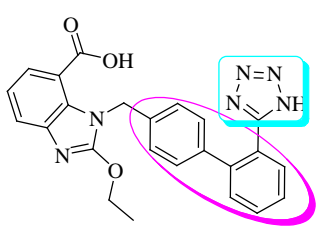

Candesartan

Figure 1. Structure of some angiotensin receptor blockers (ARB) containing biphenyl-tetrazole moiety

\section{Results and Discussion}

\section{$2.1 \quad$ Chemistry}

The $N$-alkylation of benzyl tetrazole 2a-d with 4-bromomethylbiphenyl-2-carbonitrile 1 in presence of anhydrous $\mathrm{K}_{2} \mathrm{CO}_{3}$ afforded regioisomers viz, 1,5-disubstitued 3a-d and 2,5-disubstituted tetrazoles 4a-d. The two regioisomers have been successfully separated by column chromatography using hexane and ethyl acetate (9:1) as eluent. Further, each isomer was reacted with sodium azide and triethyl amine hydrochloride (TEA.HCl) in toluene to provide the corresponding tetrazole derivatives $5 \mathrm{a}-\mathrm{d}$ and $6 \mathrm{a}-\mathrm{d}$. Ring transformation occurred when the tetrazole derivatives $5 \mathrm{a}-\mathrm{d}$ and $6 \mathrm{a}-\mathrm{d}$ were treated with acetic anhydride to yield 1,3,4-oxadiazole derivatives 7a-d and 8a-d respectively. The proposed mechanism of ring transformation of tetrazole to 1,3,4-oxadiazole [16] is shown in Scheme 2.

All the final compounds were characterized by FT-IR, ${ }^{1} \mathrm{H} \&{ }^{13} \mathrm{C}$ NMR, GC-MS and elemental analysis. In case of IR studies, compounds 3a-d and 4a-d have shown a sharp band around 2220-2238 $\mathrm{cm}^{-1}$ for a nitrile group. In ${ }^{1} \mathrm{H}$ NMR, the methyl protons of 1,3,4-oxadiazole derivatives 7a-d and 8a-d resonated as singlet in the range $\delta 2.20-2.35 \mathrm{ppm}$. All the compounds have shown two singlets in the range $\delta 4.20$ $4.30 \mathrm{ppm}$ and $\delta 5.35-5.83 \mathrm{ppm}$ for methylene protons. The aromatic protons of all the title compounds showed signals around $\delta 7.21-8.01 \mathrm{ppm}$. The methylene group in the regioisomers $3 \mathrm{a}-\mathrm{d} / 4 \mathrm{a}-\mathrm{d}, 5 \mathrm{a}-\mathrm{d} / 6 \mathrm{a}-\mathrm{d}$ and 7a-d/8a-d flanked between biphenyl group and tetrazole were distinguished based upon their chemical shifts. In case of 3a-d, 5a-d and 4-d, 7a-d, the singlet for this methylene group was upfield relative to those in $6 \mathrm{a}-\mathrm{d}$ and $8 \mathrm{a}-\mathrm{d}$ respectively where the chemical shift was downfield. For example, in case of the compounds 3a-d these methylene protons were resonated in the range 5.35-5.42 ppm whereas in case of their regioisomers 4a-d these methylene protons have appeared as a singlet in the range 5.80$5.86 \mathrm{ppm}$. Similar observation is made in case of the title compounds $5 \mathrm{a}-\mathrm{d} / 6 \mathrm{a}-\mathrm{d}$ and $7 \mathrm{a}-\mathrm{d} / 8 \mathrm{a}-\mathrm{d}$ respectively. In ${ }^{13} \mathrm{C}$ NMR spectroscopic analyses all the compounds have shown signals which are consistent with the number of magnetically nonequivalent carbon atoms. The mass spectroscopic studies showed the molecular ion peaks at their respective $\mathrm{m} / \mathrm{z}$ values.

\subsection{Single Crystal X-ray Analysis}

Single crystals of 5-\{4'-[(5-benzyl-2 $H$-tetrazol-2-yl)methyl] biphenyl-2-yl $\}$ - $1 H$-tetrazole monohydrate $6 \mathrm{a}$ were obtained using dry acetone by slow evaporation at room temperature and crystalline state is characterized by a long range, well defined three dimensional orders. The asymmetric unit of $5-\left\{4^{\prime}-[(5-\right.$ benzyl-2 $H$-tetrazol-2-yl) methyl] biphenyl -2-yl $\}-1 H$-tetrazole is shown in Figure 2 . The dihedral angle between the tetrazole $(\mathrm{C} 6 / \mathrm{N} 2 / \mathrm{N} 3-\mathrm{N} 5, \mathrm{C} 23 / \mathrm{C} 24 / \mathrm{N} 25-\mathrm{N} 27)$ rings is $69.58(1)^{\circ}$ while the terminal phenyl ring (C8-C13) has dihedral angles of 26.98 (8) ${ }^{\circ}$ and 39.75 (8) ${ }^{\circ}$ with the other benzene rings $(\mathrm{C} 15-\mathrm{C} 20$ \& $\mathrm{C} 21 / \mathrm{C} 22 / \mathrm{C} 28 / \mathrm{C} 29-\mathrm{C} 31)$. The dihedral angle for biphenyl $(\mathrm{C} 15-\mathrm{C} 20$ \& $\mathrm{C} 21 \backslash \mathrm{C} 22 \backslash \mathrm{C} 28 \backslash \cdot \cdots \mathrm{C} 31$ is $55.23(8)^{\circ}$. In the crystal, the solvent water molecule is linked to the main molecule via N27-H12 • . •

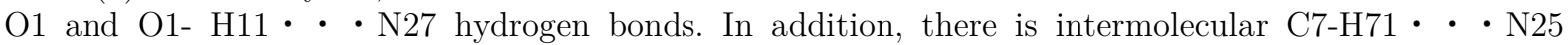
hydrogen. The structure contains $\Pi-\pi$, with a centroid-centroid $(\mathrm{Cg}(1), \mathrm{C} 6 / \mathrm{N} 2 / \mathrm{N} 3-\mathrm{N} 5)$ and $(\mathrm{Cg}(2)$, C23/24/N25-N27) distance of 3.6556 (9) A and 3.826 (1) $\mathrm{A}^{\circ}$ respectively and also $\mathrm{C}-\mathrm{H} \cdot \cdots \cdot \Pi$ interactions. In the structure, all bond lengths and angles are within normal ranges. Crystal Packing diagram is shown in Figure 3. 
<smiles>N#Cc1ccccc1-c1ccc(CBr)cc1</smiles>

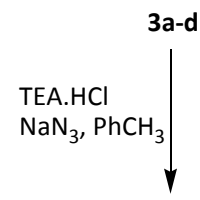<smiles>[R][X]c1nnnn1Cc1ccc(-c2ccccc2-c2nnc(C)o2)cc1</smiles>

7a-d<smiles>[R][X]c1nnnn1Cc1ccc(-c2ccccc2-c2nn[nH]n2)cc1</smiles>

5a-d<smiles>[R][X]c1nnnn1Cc1ccc(-c2ccccc2C#N)cc1</smiles><smiles>[R][X]c1nnn(Cc2ccc(-c3ccccc3C#N)cc2)n1</smiles>

a-d $\mathrm{NaN}_{3}, \mathrm{PhCH}_{3}$

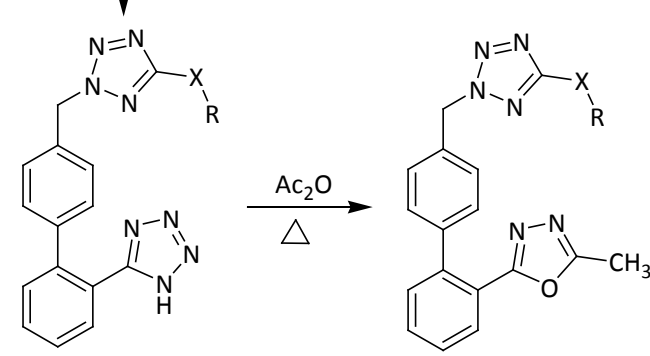

$6 a-d$

a: $\mathrm{R}=\mathrm{C}_{6} \mathrm{H}_{5}, \mathrm{X}=\mathrm{CH}_{2} ; \mathbf{b}: \mathrm{R}=\mathrm{p}-\mathrm{BrC}_{6} \mathrm{H}_{5}, \mathrm{X}=\mathrm{CH}_{2}$

c: $\mathrm{R}=\mathrm{C}_{6} \mathrm{H}_{5}, \mathrm{X}=\mathrm{CH}_{2} \mathrm{~S} ; \mathrm{d}: \mathrm{R}=\mathrm{p}-\mathrm{BrC}_{6} \mathrm{H}_{5}, \mathrm{X}=\mathrm{CH}_{2} \mathrm{~S}$

Scheme 1. Synthetic route for biphenyl derivatives

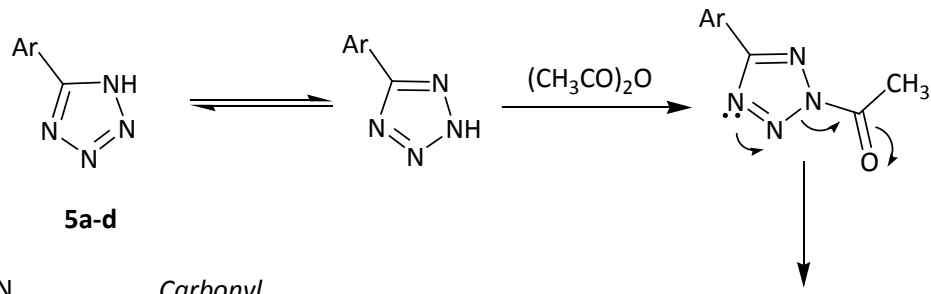<smiles>Cc1nnc([Al])o1</smiles>
insertion

$7 a-d$<smiles>[R][X]c1nnnn1Cc1ccc(-c2ccccc2C(C)C)cc1</smiles><smiles>CC(=O)N=NC[Te]</smiles>

Carbene

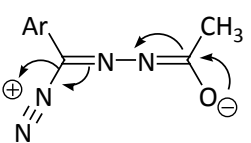

Scheme 2. Ring transformation of tetrazole to 1,3,4-oxadiazole 


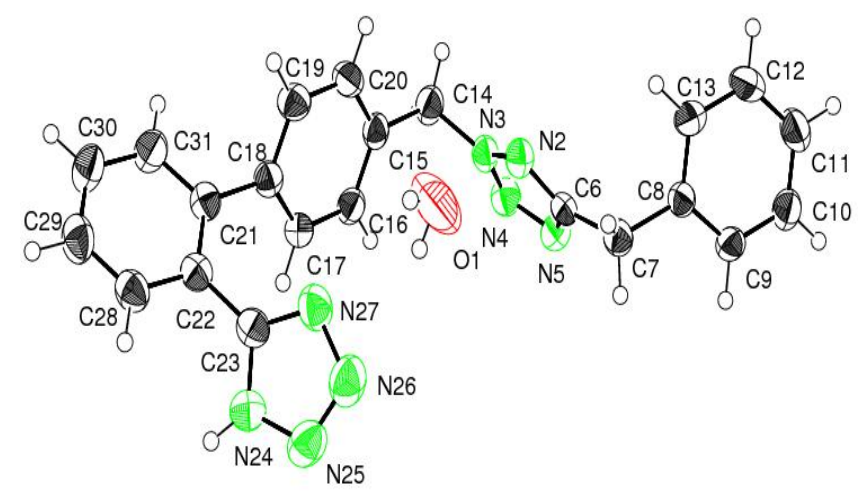

Figure 2. The molecular structure of the compound 6a (Displacement ellipsoids are drawn at the $50 \%$ probability level. Hydrogen atoms are shown as spheres of arbitrary radius)

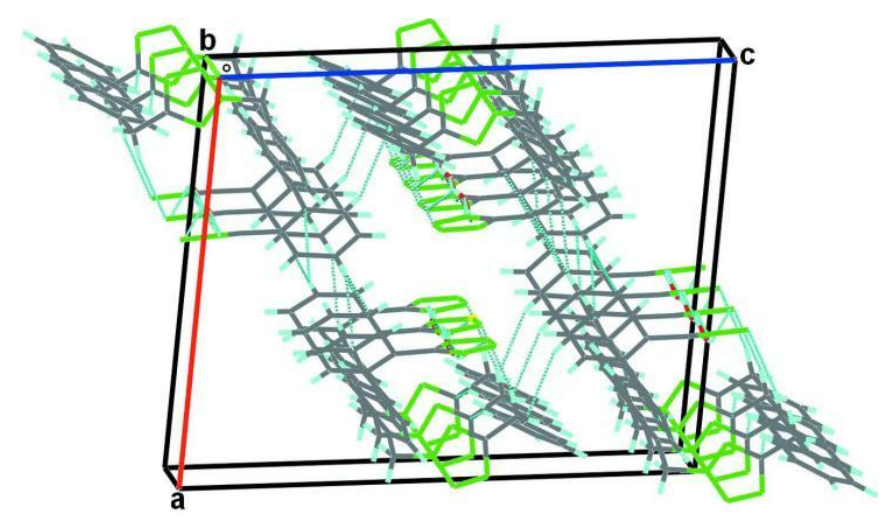

Figure 3. Molecular packing diagram of compound 6a.

\section{$2.3 \quad$ In Vitro ACE Inhibition}

The ultimate action of an angiotensin converting enzyme (ACE) inhibitor is to relax the blood vessels and lower blood pressure. ACE inhibitors block the conversion of angiotensin I to angiotensin II enzyme and thereby also inhibit the formation of angiotensin II that occurs in the kidney, blood vessels, heart, brain and adrenal glands. ACE inhibitors decrease the activity of the renin-angiotensin-aldosterone system (RAAS) which controls the variations in the blood pressure. RAAS also produces a protein named renin which is released from the kidneys, which in turn produces angiotensin that induces the adrenal gland to produce aldosterone. Aldosterone checks the balance of the reabsorption of water and conservation of sodium, with the increase in water retention and blood pressure in human body. Angiotensin II increases blood pressure by its action as a vasoconstrictive substance and steadily stimulates the secretion of aldosterone. Thus, ACE is the primary target for tacking hypertension which results in increasing cardiac output, cardiac index, stroke work, and lowering the renovascular resistance $[17,18]$. Therefore, in the present work we have made an attempt to design and synthesize the tetrazole and 1,3,4-oxadiazole derivatives of biphenyl which are the structural analogs of angiotensin II receptor antagonists (sartans) and assay for their inhibitory action of ACE.

Since these biphenyls 5a-d and 6a-d are the structural analogs of sartans, we have carried out the inhibition assay against ACE. It was observed that the compounds 6a-d exhibited good inhibition usually at higher concentration. In particular, the compound $6 \mathrm{c}$ with the phenyl sulfanyl moiety has 
shown very good inhibition at $1 \mu \mathrm{g}$ i.e., 90\%. However at $5 \mu \mathrm{g}$ and $10 \mu \mathrm{g}$ concentration the inhibition plateaus at 95\%. Compounds 5a-d showed moderate inhibition whereas the ring transformed compounds 7a-d and 8a-d are not active against the ACE. Hence, it can be concluded that tetrazole ring attached directly to biphenyl carbon is a requirement to possess activity (Table 1 and 2).

Table 1. Antihypertensive activity of 5a-d and 6a-d

\begin{tabular}{|c|c|c|c|c|c|}
\hline Samples & $\begin{array}{l}\text { Concentration } \\
(\mu \mathrm{g})\end{array}$ & $\begin{array}{l}\text { Inhibition } \\
(\%)\end{array}$ & Samples & $\begin{array}{l}\text { Concentration } \\
(\mu \mathrm{g})\end{array}$ & $\begin{array}{l}\text { Inhibition } \\
(\%)\end{array}$ \\
\hline & 10 & 50 & & 10 & 76 \\
\hline & 5 & 30 & & 5 & 43 \\
\hline & 1 & 22 & & 1 & 39 \\
\hline & 10 & 52 & & 10 & 85 \\
\hline & 5 & 29 & & 5 & 41 \\
\hline & 1 & 19 & & 1 & 18 \\
\hline & 10 & 60 & & 10 & 95 \\
\hline & 5 & 27 & & 5 & 95 \\
\hline & 1 & 15 & & 1 & 90 \\
\hline & 10 & 69 & & 10 & 88 \\
\hline & 5 & 36 & & 5 & 47 \\
\hline & 1 & 12 & & 1 & 12 \\
\hline & & & & & \\
\hline & & & Control & ---- & 63 \\
\hline
\end{tabular}

Table 2. Antihypertensive activity of 7a-d and 8a-d

Samples




(10)

\section{Conclusions}

From the above study, we reported the design and efficient synthesis of 5a-d/6a-d and further ring transformation into 7a-d/8a-d i.e., tetrazole to 1,3,4-oxadiazole ring. Diverse, albeit small libraries of these compounds were evaluated for the inhibition of ACE enzyme. From the in vitro results, it was found that some of the tetrazole derivatives $5 \mathrm{~b}, 5 \mathrm{c}$, and $5 \mathrm{~d}$ inhibited the ACE enzyme very effectively. Compounds $6 \mathrm{c}$ and $6 \mathrm{~d}$ showed the inhibition of around $90 \%$ in comparison to the control. It is interesting to note that the 1,3,4-oxadiazole derivatives $7 \mathrm{a}-\mathrm{d} / 8 \mathrm{a}-\mathrm{d}$ showed negligible activity against both the enzymes. Thus tetrazole derivatives of biphenyl $6 \mathrm{c}$ and $6 \mathrm{~d}$ can serve as lead targets for ACE inhibition in future.

\section{$4 \quad$ Experimental}

\subsection{General Procedure for the Synthesis of 4'-[(5-Aryl/Arylsulfanyl-Tetrazol) Methyl] Biphenyl-2-Carbonitrile (3a-d/4a-d)}

4-Bromomethylbiphenyl-2-carbonitrile $(\mathbf{1}, 0.01 \mathrm{M})$, benzyltetrazole $(2 \mathrm{a}-\mathrm{d}, 0.01 \mathrm{M})$, anhydrous potassium carbonate $(0.01 \mathrm{M})$ and methanol $(10 \mathrm{ml})$ were stirred in round bottom flask for $6-8 \mathrm{~h}$ at room temperature. The completion of reaction was monitored by TLC. The reaction mixture was quenched in ice cold water and the solid obtained was filtered and dried. The two regioisomers were successfully separated by column chromatography using hexane ethyl acetate (9:1) mixture as eluent.

\subsubsection{4'-[(5-Benzyl-1 $H$-tetrazol-1-yl) methyl] biphenyl-2-carbonitrile (3a)}

Pale yellow solid, mp.: $75-77^{\circ} \mathrm{C}$; IR $\left(\mathrm{KBr}, \mathrm{cm}^{-1}\right): 2229(\mathrm{CN}) ;{ }^{1} \mathrm{H}$ NMR $\left(300 \mathrm{MHz}, \mathrm{CDCl}_{3}, \delta \mathrm{ppm}\right)$ : 7.17-7.78 (13H, m, Ar-H), $5.42\left(2 \mathrm{H}, \mathrm{s}\right.$, biphenyl $\left.\mathrm{CH}_{2}\right), 4.21\left(2 \mathrm{H}, \mathrm{s}, \mathrm{CH}_{2}\right) ;{ }^{13} \mathrm{C} \mathrm{NMR}\left(75 \mathrm{MHz}, \mathrm{CDCl}_{3}\right.$, $\delta$ ppm) 154.22, 148.80, 145.28, 137.90, 135.09, 135.90, 133.90, 132.90, 132.45, 132.09, 131.00, 129.32, 127.83, 120.88, 116.99, 114.88, 47.23, 33.47; MS (m/z, $70 \mathrm{eV}): 351\left(\mathrm{M}^{+}\right), 341,295,204,192,165,91,83$; Analysis for $\mathrm{C}_{22} \mathrm{H}_{17} \mathrm{~N}_{5}$, Calculated: C 75.19, H 4.88, N 19.93. Found C: 75.25, H 4.96, N 20.05.

4.1.2 4'-\{[5-(4-Bromobenzyl)-1 $H$-tetrazol-1-yl] methyl $\}$ biphenyl-2-carbonitrile $(3 \mathrm{~b})$

Pale yellow solid, mp.: 86-87 $\mathrm{C}$; IR $\left(\mathrm{KBr}, \mathrm{cm}^{-1}\right)$ : $2232(\mathrm{CN}) ;{ }^{1} \mathrm{H}$ NMR $\left(300 \mathrm{MHz}, \mathrm{CDCl}_{3}, \delta \mathrm{ppm}\right)$ : 7.10-7.79 (12H, m, Ar-H), $5.37\left(2 \mathrm{H}, \mathrm{s}\right.$, biphenyl $\left.\mathrm{CH}_{2}\right), 4.19\left(2 \mathrm{H}, \mathrm{s}, \mathrm{CH}_{2}\right) ;{ }^{13} \mathrm{C}$ NMR $\left(75 \mathrm{MHz}, \mathrm{CDCl}_{3}, \delta\right.$ ppm): 149.80, 145.28, 137.90, 135.90, 135.09, 133.90, 132.45, 132.09, 129.32, 127.83 , 127.10, 126.58, 125.32, 120.88, 117.00, 114.38, 47.53, 33.87; MS (m/z, $70 \mathrm{eV}): 431\left(\mathrm{M}^{+}+2\right), 429\left(\mathrm{M}^{+}\right), 379,351,249,192$, $165,139,111,85,57$; Analysis for $\mathrm{C}_{22} \mathrm{H}_{16} \mathrm{~N}_{5} \mathrm{Br}$, Calculated: C 61.41, H 3.75, N 16.28. Found: C 61.49, H, 3.86, N 16.36 .

\subsubsection{4'-\{[5-(Benzylsulfanyl)- $1 H$-tetrazol-1-yl $]$ methyl $\}$ biphenyl-2-carbonitrile (3c)}

Pale yellow solid, mp.: 78-80 C; IR (KBr, $\left.\mathrm{cm}^{-1}\right) 2222(\mathrm{CN}) ;{ }^{1} \mathrm{H}$ NMR $\left(300 \mathrm{MHz}, \mathrm{CDCl}_{3}\right.$, $\left.\delta \mathrm{ppm}\right): 7.27-$ $7.75(13 \mathrm{H}, \mathrm{m}, \mathrm{Ar}-\mathrm{H}), 5.36\left(2 \mathrm{H}, \mathrm{s}\right.$, biphenyl $\left.\mathrm{CH}_{2}\right), 5.25\left(2 \mathrm{H}, \mathrm{s}, \mathrm{CH}_{2}\right) ;{ }^{13} \mathrm{C} \mathrm{NMR}\left(75 \mathrm{MHz}, \mathrm{CDCl}_{3}, \delta \mathrm{ppm}\right)$ : $154.36,145.38,132.86,132.00,131.02,130.00,129.84,129.00,128.74,128.49,127.66,127.01,126.03$, 
125.23, 119.32, 113.87, 48.61, 34.78; MS (m/z, $70 \mathrm{eV}): 383\left(\mathrm{M}^{+}\right), 204,192,177,165,117,91,83,65,40$; Analysis for $\mathrm{C}_{22} \mathrm{H}_{17} \mathrm{~N}_{5} \mathrm{~S}$, Calculated: C 68.91, H 4.47, N 18.26. Found: C 68.96, H 4.54, N 18.34.

4.1.4 4'-(\{5-[(4-Bromobenzyl)sulfanyl]-1H-tetrazol-1-yl $\}$ methyl)biphenyl-2-carbonitrile (3d)

Light brown solid, mp.: 130-32 ${ }^{\circ} \mathrm{C}$; IR $\left(\mathrm{KBr}, \mathrm{cm}^{-1}\right): 2226(\mathrm{CN}) ;{ }^{1} \mathrm{H} \mathrm{NMR}\left(300 \mathrm{MHz}, \mathrm{CDCl}_{3}, \delta \mathrm{ppm}\right)$ : 7.27-7.85 (12H, m, Ar-H), $5.34\left(2 \mathrm{H}, \mathrm{s}\right.$, biphenyl $\left.\mathrm{CH}_{2}\right), 5.21\left(2 \mathrm{H}, \mathrm{s}, \mathrm{CH}_{2}\right) ;{ }^{13} \mathrm{C} \mathrm{NMR}\left(75 \mathrm{MHz} \mathrm{CDCl}_{3}, \delta\right.$ ppm): 153.68, 145.20, 137.81, 135.07, 133.94, 132.80, 132.03, 130.99, 129.00, 128.50, 127.73, 126.25, 125.36, 122.36, 116.86, 113.87, 47.61, 37.86; MS (m/z, $70 \mathrm{eV}): 463\left(\mathrm{M}^{+}+2\right), 461\left(\mathrm{M}^{+}\right), 390,341,295,281$, 211, 193, 180, 165, 91, 65; Analysis for $\mathrm{C}_{22} \mathrm{H}_{16} \mathrm{~N}_{5}$ BrS, Calculated: C 57.15, H 3.49, N 15.15. Found: C 57.21, H 3.59, N 15.21.

4.1.5 4'-[(5-Benzyl-2H-tetrazol-2-yl)methyl]biphenyl-2-carbonitrile (4a)

White solid, mp.: $72-74^{\circ} \mathrm{C}$; IR $\left(\mathrm{KBr}, \mathrm{cm}^{-1}\right): 2224(\mathrm{CN}) ;{ }^{1} \mathrm{H}$ NMR $\left(300 \mathrm{MHz}, \mathrm{CDCl}_{3}, \delta\right.$ ppm): $7.22-7.65$ $(13 \mathrm{H}, \mathrm{m}, \mathrm{Ar}-\mathrm{H}), 5.81\left(2 \mathrm{H}, \mathrm{s}\right.$, biphenyl $\left.\mathrm{CH}_{2}\right), 4.22\left(2 \mathrm{H}, \mathrm{s}, \mathrm{CH}_{2}\right) ;{ }^{13} \mathrm{C} \mathrm{NMR}\left(75 \mathrm{MHz}, \mathrm{CDCl}_{3}, \delta \mathrm{ppm}\right)$ : $153.54,145.27,137.32,136.30,135.86,135.40,132.85,132.30,129.75,128.82,128.72,127.81,126.80$, 125.42, 124.80, 116.88, 48.33, 34.88; MS (m/z, $70 \mathrm{eV}): 351,341,295,204,192,165,91$, 65; Analysis for $\mathrm{C}_{22} \mathrm{H}_{17} \mathrm{~N}_{5}$ Calculated: C 75.19, H 4.88, N 19.93. Found: C 75.28, H 4.97, N 20.04.

4.1.6 4'-\{[5-(4-Bromobenzyl)-2H-tetrazol-2-yl $]$ methyl $\}$ biphenyl-2-carbonitrile (4b)

Light brown solid, mp.: 128-30 C; IR $\left(\mathrm{KBr}, \mathrm{cm}^{-1}\right): 2235(\mathrm{CN}) ;{ }^{1} \mathrm{H} \mathrm{NMR}\left(300 \mathrm{MHz}, \mathrm{CDCl}_{3}, \delta \mathrm{ppm}\right)$ : 7.27-7.87 (12H, m, Ar-H), $5.80\left(2 \mathrm{H}, \mathrm{s}\right.$, biphenyl $\left.\mathrm{CH}_{2}\right), 4.20\left(2 \mathrm{H}, \mathrm{s}, \mathrm{CH}_{2}\right) ;{ }^{13} \mathrm{C} \mathrm{NMR}\left(75 \mathrm{MHz}, \mathrm{CDCl}_{3}, \delta\right.$ ppm): 153.30, 145.30, 136.30, 135.70, 134.40, 132.90, 132.40, 131.80, 129.38, 128.90, 128.05, 127.71, 126.56, 125.46, 119.80, 114.80, 48.33, 34.85; MS (m/z, $70 \mathrm{eV}): 431\left(\mathrm{M}^{+}+2\right), 429\left(\mathrm{M}^{+}\right), 322,289,235,192$, 165, 91, 65; Analysis for $\mathrm{C}_{22} \mathrm{H}_{16} \mathrm{~N}_{5} \mathrm{Br}, \mathrm{C}$ 61.41, $\mathrm{H}$ 3.75, N 16.28. Found: C 61.48, H 3.86, N 16.37.

4.1.7 4'- $\{[5-(B e n z y l s u l f a n y l)-2 H$-tetrazol-2-yl $]$ methyl $\}$ biphenyl-2-carbonitrile (4c)

Pale yellow powder, mp.: 68-70 C; IR $\left(\mathrm{KBr}, \mathrm{cm}^{-1}\right): 2238(\mathrm{CN}) ;{ }^{1} \mathrm{H} \mathrm{NMR}\left(300 \mathrm{MHz}, \mathrm{CDCl}_{3}, \delta \mathrm{ppm}\right)$ : 7.25-7.68 (13H, m, Ar-H), $5.83\left(2 \mathrm{H}, \mathrm{s}\right.$, biphenyl $\left.\mathrm{CH}_{2}\right), 4.58\left(2 \mathrm{H}, \mathrm{s}, \mathrm{CH}_{2}\right) ;{ }^{13} \mathrm{C} \mathrm{NMR}\left(75 \mathrm{MHz}, \mathrm{CDCl}_{3}, \delta\right.$ ppm): 154.22, 146.17, 137.85, 136.80, 135.80, 132.56, 132.47, 130.90, 129.42, 128.99, 128.87, 128.49, 127.78, 126.33, 116.87, 113.87, 47.50, 38.08; MS (m/z, $70 \mathrm{eV}): 383,204,192,177,165,117,91,83,65,40$; Analysis for $\mathrm{C}_{22} \mathrm{H}_{17} \mathrm{~N}_{5} \mathrm{~S}$, Calculated: $\mathrm{C}$ 68.91, H 4.47, N 18.26. Found: C 69.06, H 4.54, N 18.34.

4.1.8 4'-(\{5-[(4-Bromobenzyl)sulfanyl]-2H-tetrazol-2-yl $\}$ methyl)biphenyl-2-carbonitrile (4d)

Light brown solid, mp.: 110-12 $\mathrm{C}$; IR $\left(\mathrm{KBr}, \mathrm{cm}^{-1}\right): 2229(\mathrm{CN}) ;{ }^{1} \mathrm{H}$ NMR $\left(300 \mathrm{MHz}, \mathrm{CDCl}_{3}, \delta \mathrm{ppm}\right)$ : 7.15-7.68 (12H, m, Ar-H), $5.86\left(2 \mathrm{H}\right.$, biphenyl $\left.\mathrm{CH}_{2}\right), 4.59\left(2 \mathrm{H}, \mathrm{s}, \mathrm{CH}_{2}\right) ;{ }^{13} \mathrm{C} \mathrm{NMR}\left(75 \mathrm{MHz}, \mathrm{CDCl}_{3}, \delta\right.$ ppm): $150.00,145.25,137.50,135.80,134.00,132.80,132.03,130.98,128.82,127.73,127.73,126.54$, 125.47, 122.36, 116.90, 115.10, 48.21, 37.89; MS (m/z, $70 \mathrm{eV}): 462,341,295,281,211,193,165,152,103$, 91, 65; Analysis for $\mathrm{C}_{22} \mathrm{H}_{16} \mathrm{~N}_{5} \mathrm{BrS}$, Calculated: C 57.15, H 3.49, N 15.15. Found: C 57.21, H 3.58, N 15.26.

\subsection{General Procedure for the Synthesis of 5-(Aryl/Arylsulfanyl)-1-\{[2'-(1H-Tetrazol-5-} yl) Biphenyl-4-yl] Methyl\}-1H-Tetrazole (5a-d)

A mixture of 4'-[(5-aryl/aryl-sulfanyl-1H-tetrazol-1-yl)methyl]biphenyl-2-carbonitrile 3a-d, (0.01 M), sodium azide $(0.04 \mathrm{M})$ and triethylamine hydrochloride (TEA, $0.04 \mathrm{M})$ in toluene $(20 \mathrm{ml})$ was stirred at $105-110^{\circ} \mathrm{C}$ for $48 \mathrm{~h}$. After completion of reaction, the reaction mixture was cooled to room temperature and extracted with $10 \%$ sodium hydroxide solution $(20 \mathrm{ml})$ and water. The aqueous layer was collected and dil. hydrochloric acid is added until acidic. The solid separated was filtered, dried and recrystallized using ethanol.

A similar procedure was followed to prepare 5-\{4'-[(5-aryl/arylsulfanyl)-2H-tetrazol-2-yl $)$ methyl $]$ biphenyl-2-yl\}-1H-tetrazole (6a-d) from 4'-[(5-aryl/arylsulfanyl-2H-tetrazol-2-yl) methyl] biphenyl-2carbonitrile $(4 \mathrm{a}-\mathrm{d})$.

4.2.1 5-Benzyl-1- $\{[2 '-(1 H$-tetrazol-5-yl) biphenyl-4-yl] methyl $\}-1 H$-tetrazole (5a)

Pale yellow solid, mp.: $141-43^{\circ} \mathrm{C}$; IR $\left(\mathrm{KBr}, \mathrm{cm}^{-1}\right): 1619,1556 ;{ }^{1} \mathrm{H} \mathrm{NMR}\left(300 \mathrm{MHz}, \mathrm{CDCl}_{3}, \delta \mathrm{ppm}\right)$ : 7.23-7.68 (13H, m, Ar-H), $5.41\left(2 \mathrm{H}, \mathrm{s}\right.$, biphenyl $\left.\mathrm{CH}_{2}\right), 4.20\left(2 \mathrm{H}, \mathrm{s}, \mathrm{CH}_{2}\right) ;{ }^{13} \mathrm{C} \mathrm{NMR}\left(75 \mathrm{MHz}, \mathrm{CDCl}_{3}, \delta\right.$ ppm): $150.00,148.53,137.90,135.79,131.00,130.94,129.42,129.00,128.50,128.30,128.00,127.16$, 126.82, 126.70, 125.00, 120.01, 48.11, 34.01; MS (m/z, $70 \mathrm{eV}): 394,351,289,235,192,178,165,152,91$, 44; Analysis for $\mathrm{C}_{22} \mathrm{H}_{18} \mathrm{~N}_{8}$, Calculated: $\mathrm{C} 66.99, \mathrm{H}$ 4.60, N 28.41. Found: $\mathrm{C} 67.03, \mathrm{H} 4.72, \mathrm{~N} 28.52$.

4.2.2 5-(4-Bromobenzyl)-1- $\left\{\left[2^{\prime}-(1 H\right.\right.$-tetrazol-5-yl) biphenyl-4-yl $]$ methyl $\}-1 H$-tetrazole (5b)

Pale yellow solid, mp.: $186-88^{\circ} \mathrm{C}$; IR $\left(\mathrm{KBr}, \mathrm{cm}^{-1}\right): 1617,1456 ;{ }^{1} \mathrm{H} \mathrm{NMR}\left(300 \mathrm{MHz}, \mathrm{CDCl}_{3}, \delta \mathrm{ppm}\right)$ : 7.21-7.93 (12H, m, Ar-H), $5.30\left(2 \mathrm{H}, \mathrm{s}\right.$, biphenyl $\left.\mathrm{CH}_{2}\right), 4.19\left(2 \mathrm{H}, \mathrm{s}, \mathrm{CH}_{2}\right) ;{ }^{13} \mathrm{C} \mathrm{NMR}\left(75 \mathrm{MHz}, \mathrm{CDCl}_{3}, \delta\right.$ 
ppm): 150.99, 148.50, 139.00, 138.09, 137.90, 135.28, 134.30, 132.10, 131.00, 129.54, 128.99, 128.50, 127.66, 127.03, 122.88, 116.50, 47.99, 33.99; MS (m/z, $70 \mathrm{eV}): 474\left(\mathrm{M}^{+}+2\right), 472\left(\mathrm{M}^{+}\right), 322,289,207,192$, 178, 165, 91, 44; Analysis for $\mathrm{C}_{22} \mathrm{H}_{17} \mathrm{~N}_{8} \mathrm{Br}$, Calculated: C 55.83, H 3.62, N 23.62. Found: C 55.96, H 3.70, N 23.68.

4.2.3 5-(Benzylsulfanyl)-1- $\{[2$ '-(1H-tetrazol-5-yl)biphenyl-4-yl $]$ methyl $\}-1 H$-tetrazole $(5 \mathrm{c})$

Off white solid, mp.: 140-43 ${ }^{\circ}$; IR $\left(\mathrm{KBr}, \mathrm{cm}^{-1}\right)$ 1621, 1448; ${ }^{1} \mathrm{H}$ NMR $\left(300 \mathrm{MHz}, \mathrm{CDCl}_{3}, \delta \mathrm{ppm}\right): 7.22-$ $7.73(13 \mathrm{H}, \mathrm{m}, \mathrm{Ar}-\mathrm{H}), 5.37\left(2 \mathrm{H}, \mathrm{s}\right.$, biphenyl $\left.\mathrm{CH}_{2}\right), 4.52\left(2 \mathrm{H}, \mathrm{s}, \mathrm{CH}_{2}\right) ;{ }^{13} \mathrm{C} \mathrm{NMR}\left(75 \mathrm{MHz}, \mathrm{CDCl}_{3}, \delta \mathrm{ppm}\right)$ : $151.29,149.17,137.85,136.80,135.99,135.80,132.34,132.40,130.90,129.42,128.99,128.87,128.49$, 127.68, 126.88, 118.87, 47.50, 38.08; MS (m/z, $70 \mathrm{eV}): 426,210,192,176,165,93,48$; Analysis for $\mathrm{C}_{22} \mathrm{H}_{18} \mathrm{~N}_{8} \mathrm{~S}$, Calculated: C 61.95, H 4.25, N 36.25. Found: C 62.04, H 4.31, N 26.34.

\subsubsection{5-[(4-Bromobenzyl)sulfanyl]-1-\{[2'-(1H-tetrazol-5-yl)biphenyl-4-yl]methyl $\}-1 H$ -} tetrazole $(5 \mathrm{~d})$

Pale yellow solid, mp.: $134-36^{\circ} \mathrm{C}$; IR $\left(\mathrm{KBr}, \mathrm{cm}^{-1}\right)$ : 1618, $1458 ;{ }^{1} \mathrm{H}$ NMR $\left(300 \mathrm{MHz}, \mathrm{CDCl}_{3}, \delta \mathrm{ppm}\right)$ : 7.27-7.83 (12H, m, Ar-H), $5.38\left(2 \mathrm{H}, \mathrm{s}\right.$, biphenyl $\left.\mathrm{CH}_{2}\right), 4.52\left(2 \mathrm{H}, \mathrm{s}, \mathrm{CH}_{2}\right) ;{ }^{13} \mathrm{C} \mathrm{NMR}\left(75 \mathrm{MHz}, \mathrm{CDCl}_{3}, \delta\right.$ ppm): 152.00, 150.23, 136.48, 135.89, 135.53, 133.99, 132.50, 132.00, 130.98, 130.00, 129.62, 128.40, 127.60, 126.80, 126.00, 122.40, 48.02, 37.53; MS (m/z, $70 \mathrm{eV}): 506\left(\mathrm{M}^{+}+2\right), 504\left(\mathrm{M}^{+}\right), 379,351,249,208$, 192, 165, 111, 85, 57; Analysis for $\mathrm{C}_{22} \mathrm{H}_{17} \mathrm{~N}_{8}$ BrS, Calculated: C 52.28, H 3.39, N 22.17. Found: C 52.36, H $3.45, \mathrm{~N} 22.26$.

4.2.5 5- $\{4$ '-[(5-Benzyl-2 $H$-tetrazol-2-yl) methyl]biphenyl-2-yl $\}-1 H$-tetrazole (6a)

Pale yellow solid, mp.: 212-14 ${ }^{\circ} \mathrm{C}$; IR $\left(\mathrm{KBr}, \mathrm{cm}^{-1}\right): 1622,1488 ;{ }^{1} \mathrm{H}$ NMR $\left(300 \mathrm{MHz}, \mathrm{CDCl}_{3}, \delta \mathrm{ppm}\right)$ : 7.25-7.85 (13H, m, Ar- H), $5.62\left(2 \mathrm{H}, \mathrm{s}\right.$, biphenyl $\left.\mathrm{CH}_{2}\right), 4.20\left(2 \mathrm{H}, \mathrm{s}, \mathrm{CH}_{2}\right) ;{ }^{13} \mathrm{C} \mathrm{NMR}\left(75 \mathrm{MHz}, \mathrm{CDCl}_{3}, \delta\right.$ ppm): 153.32, 145.60, 136.44, 135.86, 137.00, 135.38, 132.99, 132.38, 129.78, 128.90, 127.82, 127.00, 126.84, 126.03, 116.78, 115.68, 48.45, 34.72; MS (m/z, $70 \mathrm{eV}): 394,351,289,235,192,178,165,152,91$, 77; Analysis for $\mathrm{C}_{22} \mathrm{H}_{18} \mathrm{~N}_{8}$, Calculated: C 66.99, H 4.60, N 28.41. Found: C 67.08, H 4.74, N 28.52. 4.2.6 5-(4'-\{[5-(4-Bromobenzyl)-2H-tetrazol-2-yl $]$ methyl $\}$-biphenyl-2-yl)-1 $\boldsymbol{H}$-tetrazole $(6 \mathrm{~b})$

White solid, mp.: 158-60 $\mathrm{C}$; IR $\left(\mathrm{KBr}, \mathrm{cm}^{-1}\right): 1614,1456 ;{ }^{1} \mathrm{H}$ NMR $\left(300 \mathrm{MHz}, \mathrm{CDCl}_{3}, \delta \mathrm{ppm}\right): 7.26-7.89$ $(12 \mathrm{H}, \mathrm{m}, \mathrm{Ar}-\mathrm{H}), 5.63\left(2 \mathrm{H}, \mathrm{s}\right.$, biphenyl $\left.\mathrm{CH}_{2}\right), 4.21\left(2 \mathrm{H}, \mathrm{s}, \mathrm{CH}_{2}\right) ;{ }^{13} \mathrm{C} \mathrm{NMR}\left(75 \mathrm{MHz}, \mathrm{CDCl}_{3}, \delta \mathrm{ppm}\right)$ : $153.33,150.30,137.21,136.33,135.80,134.45,133.01,132.55,132.03,131.80,131.02,129.40,128.85$, 128.31, 125.97, 120.10, 48.36, 34.99; MS (m/z, $70 \mathrm{eV}): 474\left(\mathrm{M}^{+}+2\right), 472\left(\mathrm{M}^{+}\right), 396,273,206,193,165$, 96, 42; Analysis for $\mathrm{C}_{22} \mathrm{H}_{17} \mathrm{~N}_{8} \mathrm{Br}$, Calculated: C 55.83, H 3.62, N 23.62. Found: C 55.9, H 3.72, N 23.69. 4.2.7 5-(4'-\{[5-(Benzylsulfanyl)-2 $\boldsymbol{H}$-tetrazol-2-yl $]$ methyl $\}$ biphenyl-2-yl)-1 $\boldsymbol{H}$-tetrazole (6c)

Pale yellow solid, mp.: 131-33 ${ }^{\circ} \mathrm{C}$; IR $\left(\mathrm{KBr}, \mathrm{cm}^{-1}\right)$ : 1616, $1457 ;{ }^{1} \mathrm{H} \mathrm{NMR}\left(300 \mathrm{MHz}, \mathrm{CDCl}_{3}, \delta \mathrm{ppm}\right)$ : 7.22-7.80 (13H, m, Ar-H), $5.40\left(2 \mathrm{H}, \mathrm{s}\right.$, biphenyl $\left.\mathrm{CH}_{2}\right), 4.25\left(2 \mathrm{H}, \mathrm{s}, \mathrm{CH}_{2}\right)$, biphenyl $\left.\mathrm{CH}_{2}\right), 4.22(2 \mathrm{H}, \mathrm{s}$, $\left.\mathrm{CH}_{2}\right) ;{ }^{13} \mathrm{C} \mathrm{NMR}\left(75 \mathrm{MHz}, \mathrm{CDCl}_{3}, \delta \mathrm{ppm}\right): 154.22,146.17,137.85,136.80,135.80,132.45,132.40,130.90$, 129.42, 128.87, 128.01, 127.68, 126.12, 125.87, 116.87, 113.87, 47.50, 38.08; MS (m/z, 70 eV): 426, 204, 192, 177, 165, 91, 83; Analysis for $\mathrm{C}_{22} \mathrm{H}_{18} \mathrm{~N}_{8} \mathrm{~S}$, Calculated: C 61.95, H 4.25, N 36.25. Found: C 62.04, H 4.36, N 26.29

\subsubsection{5-[4'-(\{5-[(4-Bromobenzyl)sulfanyl]-2H-tetrazol-2-yl $\}$ methyl $)$ biphenyl-2-yl $]-1 H$ -} tetrazole $(6 \mathrm{~d})$

Pale yellow solid, mp.: 154-56 ${ }^{\circ} \mathrm{C}$; IR $\left(\mathrm{KBr}, \mathrm{cm}^{-1}\right): 1617,1461 ;{ }^{1} \mathrm{H}$ NMR $\left(300 \mathrm{MHz}, \mathrm{CDCl}_{3}, \delta \mathrm{ppm}\right)$ : 7.29-7.88 (12H, m, Ar-H), $5.90\left(2 \mathrm{H}, \mathrm{s}\right.$, biphenyl $\left.\mathrm{CH}_{2}\right), 4.23\left(2 \mathrm{H}, \mathrm{s}, \mathrm{CH}_{2}\right) ;{ }^{13} \mathrm{C} \mathrm{NMR}\left(75 \mathrm{MHz}, \mathrm{CDCl}_{3}, \delta\right.$ ppm):152.00, 150.23, 137.51, 136.48, 135.89, 135.53, 133.99, 132.00, 130.98, 128.99, 128.40, 127.40, 127.00, 126.85, 126.00, 122.40, 48.22, 37.72; MS (m/z, $70 \mathrm{eV}): 506\left(\mathrm{M}^{+}+2\right), 504\left(\mathrm{M}^{+}\right), 379,351,249,208,192$, 165, 111, 85, 57; Analysis for $\mathrm{C}_{22} \mathrm{H}_{17} \mathrm{~N}_{8} \mathrm{BrS}$, Calculated: C 52.28, H 3.39, N 22.17. Found: C 52.35, H 3.46, N 22.28.

4.3 General Procedure for the Synthesis of 5-(Aryl/Arylsulfanyl)-\{[2'-(5-Methyl-1,3,4Oxadiazol-2-yl)Biphenyl-4-yl]Methyl\}-1H-Tetrazole (7a-d)

5-(Aryl/arylsulfanyl)-1-\{[2'-(1H-tetrazol-5-yl) biphenyl-4-yl $]$ methyl $\}-1 H$-tetrazole 5a-d, 1.00gm) was heated with acetic anhydride $(10 \mathrm{ml})$ on a water bath for $2 \mathrm{~h}$. The reaction was monitored by TLC using hexane/ethyl acetate. Then the reaction mixture was poured into ice cold water. The solid separated was filtered, washed with water and dried. Recrystallization was carried using ethanol. Similarly, 5-aryl/arylsulfanyl - $\{[2$ '-(5 methyl-1,3,4 -oxadiazole-2-yl) biphenyl-4-yl $]$ methyl $\}$-21 $H$-tetrazole $8 \mathrm{a}-\mathrm{d}$ was synthesized from 5-\{4'-[(5-aryl/arylsulfanyl)-2H-tetrazol-2-yl)methyl]biphenyl-2-yl $\}-1 H$ - 
tetrazole $6 \mathrm{a}-\mathrm{d}$.

4.3.1 5-Benzyl- $\{[2$ '-(5-methyl-1,3,4-oxadiazol-2-yl)biphenyl-4-yl $]$ methyl $\}$ - $H$ H-tetrazole (7a)

Pale yellow solid, mp: $131-33^{\circ} \mathrm{C}$; IR $\left(\mathrm{KBr}, \mathrm{cm}^{-1}\right): 1616,1457 ;{ }^{1} \mathrm{H}$ NMR $\left(300 \mathrm{MHz}, \mathrm{CDCl}_{3}, \delta \mathrm{ppm}\right)$ : 7.22-7.80 (13H, m, Ar-H), $5.40\left(2 \mathrm{H}, \mathrm{s}\right.$, biphenyl $\left.\mathrm{CH}_{2}\right), 4.25\left(2 \mathrm{H}, \mathrm{s}, \mathrm{CH}_{2}\right), 2.34\left(3 \mathrm{H}, \mathrm{s}, \mathrm{CH}_{3}\right) ;{ }^{13} \mathrm{C}$ NMR $(75$ $\left.\mathrm{MHz}, \mathrm{CDCl}_{3}, \delta \mathrm{ppm}\right): 164.54,159.90,148.60,139.89,137.69,135.70,132.29,130.90,130.79,129.40$, 128.97, 128.50, 127.76, 127.47, 126.39, 125.00, 119.66, 47.99, 33.39, 15.01; MS (m/z, $70 \mathrm{eV}): 408,392$, 351, 249, 192, 165, 139, 71, 43; Analysis for $\mathrm{C}_{24} \mathrm{H}_{20} \mathrm{~N}_{6} \mathrm{O}$, Calculated: C 70.57, H 4.94, N 20.58. Found: C 70.67, H 5.04, N 20.64.

4.3.2 5-(4-Bromobenzyl)- $\{[2$ '-(5-methyl-1,3,4-oxadiazol-2-yl)biphenyl-4-yl $]$ methyl $\}-1 H$ tetrazole $(7 \mathrm{~b})$

Pale yellow solid, mp.: 165-67 $\mathrm{C}$; IR $\left(\mathrm{KBr}, \mathrm{cm}^{-1}\right)$ : 1623, $1499 ;{ }^{1} \mathrm{H}$ NMR $\left(300 \mathrm{MHz}, \mathrm{CDCl}_{3}, \delta \mathrm{ppm}\right)$ : 7.15-7.99 (12H, m, Ar-H); $5.40\left(2 \mathrm{H}, \mathrm{s}\right.$, biphenyl $\left.\mathrm{CH}_{2}\right), 4.25\left(2 \mathrm{H}, \mathrm{s}, \mathrm{CH}_{2}\right), 2.35\left(3 \mathrm{H}, \mathrm{s}, \mathrm{CH}_{3}\right) ;{ }^{13} \mathrm{C}$ NMR $(75$ $\left.\mathrm{MHz}, \mathrm{CDCl}_{3}, \delta \mathrm{ppm}\right): 164.80,160.88,145.66,139.40,138.00,137.50,135.80,133.23,133.00,134.25$, 132.10, 131.20, 128.55, 127.03, 126.85, 125.66, 122.08, 48.02, 34.09, 15.23; MS (m/z, $70 \mathrm{eV}): 488\left(\mathrm{M}^{+}+2\right)$, $486\left(\mathrm{M}^{+}\right), 382,235,205,192,165,91,65$; Analysis for $\mathrm{C}_{24} \mathrm{H}_{19} \mathrm{~N}_{6} \mathrm{BrO}$, Calculated: $\mathrm{C} 59.19, \mathrm{H} \mathrm{3.93,} \mathrm{N}$ 17.24. Found: C 59.26, H 4.05, N 17.38.

\subsubsection{5-(Benzylsulfanyl)-\{[2'-(5-methyl-1,3,4-oxadiazol-2-yl)biphenyl-4-yl $]$ methyl $\}-1 H$ -} tetrazole $(7 \mathrm{c})$

Yellow solid, mp.: 88-90 C; IR (KBr, cm $\left.{ }^{-1}\right): 1620,1485 ;{ }^{1} \mathrm{H} \mathrm{NMR}\left(300 \mathrm{MHz}, \mathrm{CDCl}_{3}, \delta \mathrm{ppm}\right): 7.08-7.80$ $(13 \mathrm{H}, \mathrm{m}, \mathrm{Ar}-\mathrm{H}), 5.35\left(2 \mathrm{H}, \mathrm{s}\right.$, biphenyl $\left.\mathrm{CH}_{2}\right), 4.45\left(2 \mathrm{H}, \mathrm{s}, \mathrm{CH}_{2}\right), 2.25\left(3 \mathrm{H}, \mathrm{s}, \mathrm{CH}_{3}\right) ;{ }^{13} \mathrm{C} \mathrm{NMR}(75 \mathrm{MHz}$, $\left.\mathrm{CDCl}_{3}, \delta \mathrm{ppm}\right): 163.60,159.90,153.59,139.22,137.30,136.80,135.76,132.99,132.30,130.80,130.00$, 129.31, 128.88, 128.48, 127.75, 127.58, 126.68, 48.68, 37.88, 16.02; MS (m/z, $70 \mathrm{eV}): 440,426,379,249$, 192, 165, 85, 43; Analysis for $\mathrm{C}_{24} \mathrm{H}_{20} \mathrm{~N}_{6} \mathrm{SO}$, Calculated: C 65.44, H 4.58, N 19.08. Found: C 65.57, H 4.64, $\mathrm{N} 19.18$

\subsubsection{5-[4-(Bromobenzyl)-sulfanyl]-\{[2'-(5-methyl-1,3,4-oxadiazol-2-yl)biphenyl-4} yl]methyl $\}-1 H$-tetrazole $(7 \mathrm{~d})$

Pale yellow solid, mp.: $127-29^{\circ} \mathrm{C}$; IR $\left(\mathrm{KBr}, \mathrm{cm}^{-1}\right): 1619,1466 ;{ }^{1} \mathrm{H} \mathrm{NMR}\left(300 \mathrm{MHz}, \mathrm{CDCl}_{3}, \delta \mathrm{ppm}\right)$ : 7.03-7.60 (12H, m, Ar-H), $5.25\left(2 \mathrm{H}, \mathrm{s}\right.$, biphenyl $\left.\mathrm{CH}_{2}\right), 4.15\left(2 \mathrm{H}, \mathrm{s}, \mathrm{CH}_{2}\right), 2.15\left(3 \mathrm{H}, \mathrm{s}, \mathrm{CH}_{3}\right) ;{ }^{13} \mathrm{C}$ NMR $(75$ $\left.\mathrm{MHz}, \mathrm{CDCl}_{3}, \delta \mathrm{ppm}\right): 169.80,163.60,159.94,139.50,137.01,135.79,133.94,132.11,131.03,130.74$, 128.82, 128.62, 128.03, 127.76, 126.69, 126.39, 125.36, 48.00, 38.03, 15.00; MS (m/z, $70 \mathrm{eV}): 520\left(\mathrm{M}^{+}+2\right)$, $518\left(\mathrm{M}^{+}\right), 426,379,351,249,192,165,111,85,71$; Analysis for $\mathrm{C}_{24} \mathrm{H}_{19} \mathrm{~N}_{6} \mathrm{BrSO}$, Calculated: $\mathrm{C} 55.50, \mathrm{H}$ 3.69, N 16.18. Found: C 55.58, H 3.76, N 16.25 .

4.3.5 5-Benzyl- $\{[2 '-(5-m e t h y l-1,3,4-o x a d i a z o l-2-y l) b i p h e n y l-4-y l]$ methyl $\}$-2H-tetrazole (8a)

Pale yellow solid, mp.: $65-70^{\circ} \mathrm{C}$; IR (KBr, $\left.\mathrm{cm}^{-1}\right)$ 1616, $1559 ;{ }^{1} \mathrm{H}$ NMR $\left(300 \mathrm{MHz}, \mathrm{CDCl}_{3}, \delta \mathrm{ppm}\right): 7.27-$ $7.68(13 \mathrm{H}, \mathrm{m}, \mathrm{Ar}-\mathrm{H}), 5.91\left(2 \mathrm{H}, \mathrm{s}\right.$, biphenyl $\left.\mathrm{CH}_{2}\right), 4.23\left(2 \mathrm{H}, \mathrm{s}, \mathrm{CH}_{2}\right), 2.24\left(3 \mathrm{H}, \mathrm{s}, \mathrm{CH}_{3}\right) ;{ }^{13} \mathrm{C} \mathrm{NMR}(75$ $\left.\mathrm{MHz}, \mathrm{CDCl}_{3}, \delta \mathrm{ppm}\right): 164.60,159.94,148.60,139.99,137.70,135.69,132.29,131.00,130.79,129.38$, 128.97, 128.50, 127.76, 127.47, 126.39, 125.59, 120.89, 47.97, 33.96, 16.00; MS (m/z, 70 eV): 408, 396, 351, 273, 246, 206, 193, 177, 96, 42; Analysis for $\mathrm{C}_{24} \mathrm{H}_{20} \mathrm{~N}_{6} \mathrm{O}$, Calculated: C 70.57, H 4.94, N 20.58. Found: C $70.56, \mathrm{H} 4.95, \mathrm{~N} 20.57$.

\subsubsection{5-(4-Bromobenzyl)- $\{[2$ '-(5-methyl-1,3,4-oxadiazol-2-yl)biphenyl-4-yl $]$ methyl $\}-2 H$ - tetrazole $(8 \mathrm{~b})$}

Pale yellow solid, mp.: 138-40 C; IR $\left(\mathrm{KBr}, \mathrm{cm}^{-1}\right)$ : 1622, $1552 ;{ }^{1} \mathrm{H} \mathrm{NMR}\left(300 \mathrm{MHz}, \mathrm{CDCl}_{3}, \delta \mathrm{ppm}\right)$ : 7.24-7.68 (12H, m, Ar-H), $5.91\left(2 \mathrm{H}, \mathrm{s}\right.$, biphenyl, $\left.\mathrm{CH}_{2}\right), 4.23\left(2 \mathrm{H}, \mathrm{s}, \mathrm{CH}_{2}\right), 2.37\left(3 \mathrm{H}, \mathrm{s}, \mathrm{CH}_{3}\right) ;{ }^{13} \mathrm{C} \mathrm{NMR}$ (75 MHz, $\left.\mathrm{CDCl}_{3}, \delta \mathrm{ppm}\right):$ 164.36, 159.94, 138.01, 137.80, 137.00, 134.20, 133.60, 132.64, 132.12, 131.79, 129.32, 128.82, 128.62, 127.32, 126.69, 125.33, 119.74, 49.02, 34.67 15.04; MS (m/z, $70 \mathrm{eV}): 488\left(\mathrm{M}^{+}+2\right)$, $486\left(\mathrm{M}^{+}\right)$, 322, 289, 207, 192, 165, 91, 64; Analysis for $\mathrm{C}_{24} \mathrm{H}_{19} \mathrm{~N}_{6} \mathrm{BrO}$, Calculated: C 59.19, $\mathrm{H} 3.93, \mathrm{~N}$ 17.24. Found: C 59.28, H 4.06, N 17.32.

\subsubsection{5-(Benzylsulfanyl)-\{[2'-(5-methyl-1,3,4-oxadiazol-2-yl)biphenyl-4-yl $]$ methyl $\}-2 H$ - tetrazole $(8 \mathrm{c})$}

Yellow solid, mp.: 88-90 $\mathrm{C}$; IR (KBr, cm $\left.{ }^{-1}\right): 1669,1552 ;{ }^{1} \mathrm{H}$ NMR (300 MHz, $\mathrm{CDCl}_{3}, \delta$ ppm): 7.08-7.80 $(13 \mathrm{H}, \mathrm{m}, \mathrm{Ar}-\mathrm{H}) 5.91\left(2 \mathrm{H}, \mathrm{s}\right.$, biphenyl $\left.\mathrm{CH}_{2}\right), 4.45\left(2 \mathrm{H}, \mathrm{s}, \mathrm{CH}_{2}\right), 2.25\left(3 \mathrm{H}, \mathrm{s}, \mathrm{CH}_{3}\right) ;{ }^{13} \mathrm{C} \mathrm{NMR}(75 \mathrm{MHz}$, $\left.\mathrm{CDCl}_{3}, \delta \mathrm{ppm}\right): 163.60,159.94,148.60,139.99,139.01,137.70,135.79,132.29,130.90,130.79,129.88$, 129.38, 128.97, 128.62, 127.76, 126.31, 125.63, 48.09, 38.39, 16.03; MS (m/z, $70 \mathrm{eV}): 440,418,410,192$, 175, 165, 95, 48; Analysis for $\mathrm{C}_{24} \mathrm{H}_{20} \mathrm{~N}_{6} \mathrm{SO}$, Calculated: C 65.44, H 4.58, N 19.08. Found: C 65.52, H 4.62, N 19.14. 


\subsubsection{5-[4-(Bromobenzyl) sulfanyl]-\{[2'-(5-methyl-1,3,4-oxadiazol-2-yl)biphenyl-4-yl $]$ methyl $\}-2 H$-tetrazole $(8 d)$}

Pale yellow solid, mp.: $127-29^{\circ} \mathrm{C}$; IR $\left(\mathrm{KBr}, \mathrm{cm}^{-1}\right)$ : 1620,$1453 ;{ }^{1} \mathrm{H}$ NMR $\left(300 \mathrm{MHz}, \mathrm{CDCl}_{3}, \delta \mathrm{ppm}\right)$ : 7.24-7.89 (12H, m, Ar-H), $5.90\left(2 \mathrm{H}, \mathrm{s}\right.$, biphenyl $\left.\mathrm{CH}_{2}\right), 4.15\left(2 \mathrm{H}, \mathrm{s}, \mathrm{CH}_{2}\right), 2.15\left(3 \mathrm{H}, \mathrm{s}, \mathrm{CH}_{3}\right) ;{ }^{13} \mathrm{C}$ NMR $(75$ $\mathrm{MHz}, \mathrm{CDCl}_{3}$, $\left.\delta \mathrm{ppm}\right): 163.60,159.94,139.50,136.32,135.79,133.94,132.69,132.11,131.03,130.74$, 129.00, 128.82, 128.62, 128.38, 127.76, 126.69, 126.39, 48.00, 38.03, 15.12; MS (m/z, $70 \mathrm{eV}): 520\left(\mathrm{M}^{+}+2\right)$, $518\left(\mathrm{M}^{+}\right), 426,379,351,249,192,165,111,85,71$; Analysis for $\mathrm{C}_{24} \mathrm{H}_{19} \mathrm{~N}_{6} \mathrm{BrSO}$, Calculated: C 55.50, H 3.69, N 16.18. Found: C 55.59, H 3.76, N 16.24.

\section{Pharmacological Evaluation}

\subsection{ACE Inhibition Assay}

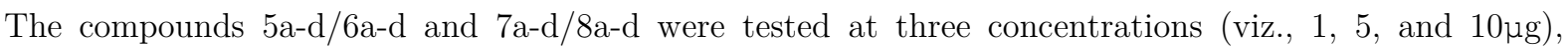
dissolved in assay buffer $[10 \mathrm{mM}$ HEPES buffer containing $\mathrm{NaCl}(0.3 \mathrm{M})$ and zinc sulphate $(10 \mu \mathrm{M})$ containing kidney cortex plasma membranes $(20 \mu \mathrm{l}$, source of ACE] and Hippuryl-His-Leu $(1 \mathrm{mM})$ as substrate. The compounds were incubated with the enzyme for $10 \mathrm{~min}$ at $37^{\circ} \mathrm{C}$. The substrate was then added and incubated for $45 \mathrm{~min}$ at $37^{\circ} \mathrm{C}$. The reaction was terminated by the addition of $\mathrm{HCl}(1 \mathrm{M})$. The yellow colour developed by the addition of pyridine $(100 \mu \mathrm{l})$ and benzene sulphonylchloride $(50 \mu \mathrm{l})$ was measured at $410 \mathrm{~nm}$ in ELISA Plate Reader (iMARK, BIORAD). Compounds with inhibitory potential block the substrate availability to the enzyme and thereby cause enzyme inhibition leading to no formation of yellow colour. The inhibition was represented in the form of percentage over control. Captopril, a known ACE inhibitor is used as the standard [19, 20].

Acknowledgments. The authors would like to thank the USIC authorities for their help in carrying out the spectral characterization (FT-IR, 1H NMR, GC-MS) and NMR Research Centre, IISc, Bangalore for 13C NMR spectral analyses. We acknowledge the assistance of Biogenics Ltd., Hubballi, Karnataka, India for carrying out the pharmacological assays. The authors acknowledge UGC, New Delhi, Govt. of India for their financial assistance vide letter No. 37-248/2009 (SR) and DSA program vide No.F-540/2/DSA/2013 (SAP-I). One of the authors (GYM) thanks the UGC, New Delhi for Junior Research Fellowship.

\section{References}

1. B. M. Y. Cheung and C. Li, "Diabetes and Hypertension: Is There a Common Metabolic Pathway?" Current Atherosclerosis Reports, vol. 14, no. 2, pp. 160-166, 2012.

2. L. Paulis, U.M. Steckelings, T. Unger, "Key advances in antihypertensive treatment," Nature Reviews Cardiology, vol. 9, no. pp. 276-285, 2012.

3. R. D. Larsen, A. O. King, C.Y. Chen, E.G. Corley, B.S. Foster, F.E. Roberts, C. Yang, D.R. Lieberman, R.A. Reamer, "Efficient synthesis of Losartan, a non-peptide angiotensin II receptor antagonist," Journal of Organic Chemistry, vol. 59, no. 21, pp. 6391-6394, 1994.

4. G. Wang, B. Sun, C. huPeng, "An improved synthesis of Valsartan," Organic Process Research and Development, vol. 15, no. 5, pp. 986-988, 2011.

5. M. P. Makkay, T. Mezei, G. Simig, "New Practical Synthesis of the Key Intermediate of Candesartan," Organic Process Research and Development, vol. 11, no. 3, pp. 490-493, 2007.

6. R. J. Herr, "5-Substituted-1H-tetrazoles as Carboxylic Acid Isosteres: Medicinal Chemistry and Synthetic Methods," Bioorganic Medicinal Chemistry, vol. 10, no. 11, pp. 3379-3393, 2002.

7. T. Mavromoustakos, A. Kolocouris, M. Zervou, P. Roumelioti, J. Matsoukas, R. Weisemann, "An effort to understand the molecular basis of hypertension through the study of conformational analysis of losartan and sarmesin using a combination of nuclear magnetic resonance spectroscopy and theoretical calculations," Journal of Medicinal Chemistry, vol. 42, no. 10, pp. 1714-1722, 1999.

8. L. V. Myznikov, A. Hrabalek, G. I. Koldobskii, "Drugs in tetrazole series," Chemistry of Heterocyclic Compounds., vol. 43, no. 1, pp. 1-9, 2007. 
9. P. B. Mohite, R. B. Pandhare, S. G. Khanage, V. H. Bhaskar, "Synthesis and anti-inflammatory activity of some 5-phenyl-1-(acyl)-1,2,3,4-tetrazole," Journal of Pharmacy Research, vol. 3, no. 1, pp. 43-46, 2010.

10. a) J. H. Toney, P. M. D. Fitzgerald, N.S. Grover, S. H. Olson, W. J. May, J. G. Sundelof, D. E. Venderwall, K. A. Cleary, S. K. Grant, W. J. K. Kozarich, J. W. Pompliano, G. G. Hammond, "Antibiotic sensitization using biphenyl tetrazoles as potent inhibitors of Bacteroidesfragilismetallo- $\beta$-lactamase," Chemistry and Biology, vol. 5, no. 4, pp. 185-196, 1998. b) G. Ortar, E, Morera, L De petrocillis, A, Ligresti, A. S. Moriello, L. Morera, M. Nalli, R. Ragno, A. Pirolli, V. D. Marzo, "Biaryl tetrazoles as inhibitors of endocannabinoid metabolism: Modulation at N-portion and distal phenyl ring", European Journal of Medicinal Chemistry, vol. 63, pp. 118-132.

11. R. A. F. Sherif, H. M.A. Ashour, Abd El Razik, E. A. Fattah, El-Din N. Nagwa, "Azole antimicrobial pharmacophore-based tetrazoles: synthesis and biological evaluation as potential antimicrobial and anticonvulsant agents," Bioorganic Medicinal Chemistry, vol. 17, no. 6, pp. 2410-2422, 2009.

12. J. P. Raval, T. N. Akhaja, J. M. Dhaval, K. N. Myangar, N. H. Patel, "Synthesis and in vitro antibacterial activity of new oxoethylthio-1,3,4-oxadiazole derivatives," Journal of Saudi Chemical Society, vol. 18, no. 2, pp. 101-106, 2014.

13. P. R. Kagthara, N. S. Shah, R. K. Doshi, H. H. Parekh, "Synthesis of 2,5-disubstituted-1,3,4-oxadiazoles as biologically active heterocycles," Indian Journal of Chemistry, vol. 38B, no. 5, pp. 572-576, 1999.

14. M. Amir, S. A. Javed, H. Kumar, "Synthesis of some 1,3,4-oxadiazole derivatives as potential anti-inflammatory agents," Indian Journal of Chemistry, vol. 46B, no.6, pp. 1014-1019, 2007.

15. R. Franski, "Biological activities of the compounds bearing 1,3,4-oxa-(thia)-diazole ring," Asian Journal of Chemistry, vol. 17, no. 4, pp. 2063- 2075, 2005.

16. G. I. Koldobski, V. A. Ostrovski, V. S. Popavski, "Advances in the Chemistry of tetrazoles," Chemistry of Heterocyclic Compounds, vol. 17, no. 10, pp. 965-989, 1981.

17. C. Haslett, E. R. Chilvers, J. A. Hunter, N. A. Boon, "Principles and practice of medicine," Livingstone, Church Hill, 1999.

18. S. K. Chaudhuri, "Concise Medical Physiology," New Central Book agency (P) Ltd., Calcutta, 1993.

19. V. K. Jimsheena, "Colorimetric, high-throughput assay for screening Angiotensin I converting enzyme inhibitors," Analytical Chemistry, vol. 81, no. 22, pp. 9388-9394, 2009.

20. N. M. Hooper, A. J. Turner, "Isolation of two differentially glycosylated forms of peptidyl-dipeptidase A (Angiotensin Converting Enzyme) from pig brain: a re-evaluation of their role in neuropeptide metabolism," Biochemical Journal, vol. 241, no. 3, pp. 625-633, 1987. 